\title{
LA LEGA "NAZIONALE" DI SALVINI ALLA CONQUISTA ELETTORALE DEL MERIDIONE
}

di Roberto De LUCA e DOMENICO FRUNCILLO 
Il saggio è frutto di un lavoro comune di progettazione, ricerca, analisi e scrittura. Ai soli fini formali i parr. 3, 4, 6 e 7 sono attribuibili a $R$. De Luca e i parr. 1, 2, 5 e 8 sono attribuibili a D. Fruncillo.

\section{The Salvini's "national" Leauge to the electoral conquest of the South}

Abstract - In the last European elections the League became the first party in Italy also because, for the first time, it collected a large number of votes in the South, exceeding the $20 \%$ of votes in the Southern italian regions. In this article we try to evaluate whether that success is temporary or if it will consolidate over time. In other words, the central question is whether the vote to the Leauge party in the South of Italy is weak and volatile or it represents the first moment of its establishment also in these areas of the Country. In details, we try to verify if the success of the Leauge party in the South of Italy can be related to traditional aspects of the parties' organization, such as the territorial root and the recruitment of political personnel who obtain in the electoral competition personal votes which improve the success of the list. The analysis was conducted through out macro and micro analysis tools. The analysis of the preference vote distribution highlights the electoral contribution offered by all the candidates to the European Parliament by region and by size of the municipalities. The description of some emblematic local cases describes the dynamics through which some local candidates for the European Parliament have contributed to increase the political consensus of the League party in the South of Italy.

Keywords: Salvini Leauge, South of Italy, Preference vote, clientelism, big electors 


\section{Premessa}

In un lasso di tempo relativamente breve nel nostro Paese si sono formati e si sono rapidamente eclissati, o addirittura dissolti, numerosi partiti. Il panorama politico attuale, infatti, è significativamente diverso da quello che poteva essere osservato negli anni Ottanta. Anche le formazioni politiche che sono nate a seguito della scomposizione e ricomposizione dei vecchi partiti hanno una fisionomia piuttosto diversa da quella dei loro ascendenti.

La Lega è il partito più vecchio tra quelli che attualmente si propongono al giudizio degli elettori in Italia e tuttavia ha una vita breve, se paragonata a quella dei grandi partiti di massa che hanno dominato la scena politica della maggior parte dei paesi democratici. La Lega, che si era affacciata sulla scena politica nazionale negli ultimi anni della cosiddetta Prima repubblica, ha superato il traumatico avvicendamento della leadership nazionale provocato anche dalle inchieste giudiziarie sull'utilizzo dei rimborsi elettorali e, negli ultimi anni, sembra sia riuscita a ribaltare il ridimensionamento elettorale che ne era seguito.

Nel corso della sua relativamente breve vicenda politica la Lega è cambiata. Non ci riferiamo qui ai rapporti con gli altri soggetti politici, alla sua collocazione nello spazio politico del nostro paese, ma piuttosto ai cambiamenti che riguardano la Lega in quanto partito. Sotto questo profilo l'attenzione dei mezzi di comunicazione e, più in generale, degli osservatori si è spesso concentrata su tre aspetti: l'organizzazione, la cultura politica e l'insediamento elettorale. Sulla scorta di alcuni studi potremmo descrivere sinteticamente la trasformazione della Lega che da partito etno-regionalista, strutturato e organizzato nelle comunità locali del nord (Diamanti 1993, 2003, 2009; Passarelli e Tuorto 2012) sembra essere diventato partito sovranista che coltiva l'ambizione di insediarsi in tutto il Paese (Passarelli e Tuorto 2018; Brunazzo e Roux 2013)). In questo contributo ci occuperemo dell'insediamento elettorale a sud del Garigliano che, ovviamente, ha a che fare con la proposta-politico programmatica e con la sua organizzazione. Per meglio dire è possibile che l'aumento del patrimonio elettorale in aree diverse da quelle in cui essa aveva visto la luce, va collegato al cambiamento di enfasi rispetto ad alcuni temi, ma anche alle nuove strategie organizzative sperimentate con l'obiettivo di incrementare e stabilizzare il seguito elettorale.

È stato segnalato e documentato (Passarelli e Tuorto 2018) che la proposta politica della Lega ha ancora come preoccupazione fondamentale la "questione settentrionale". Tuttavia questa riflessione prende avvio dalla constatazione che alle ultime due consultazioni nazionali - le elezioni 
politiche ed europee - la Lega è riuscita a raccogliere anche nelle regioni meridionali un consistente numero di consensi. La domanda è se la performance della Lega alle ultime europee debba essere collegato esclusivamente ad elementi contingenti come l'appeal di Salvini o possa essere anche la manifestazione di un più stabile, seppure inziale, insediamento politico ed organizzativo.

Nella discussione pubblica il successo della Lega nel Mezzogiorno è stato attribuito alla personalità di Salvini, alla sua capacità di proporre temi a cui meridionali sarebbero sensibili e di sviluppare una efficace campagna di comunicazione su tali questioni. La nostra ipotesi generale è che questo successo sia in relazione con i mutamenti che hanno interessato la Lega negli ultimi anni. Questi cambiamenti hanno riguardato certamente la leadership del partito, che ha caratteristiche molto diverse da quella precedente (Passarelli e Tuorto 2018), le modalità e i contenuti della comunicazione, e più in generale, le strategie elettorali che hanno allargato il target del partito (Brunazzo e Roux 2013). Tuttavia la nostra attenzione si soffermerà su un aspetto specifico e, fino a questo momento, trascurato nelle valutazioni degli osservatori. Noi ipotizziamo che il successo della Lega alle elezioni europee possa essere posto in relazione anche con il reclutamento di personale politico "locale" che sia capace di affrontare la competizione elettorale e sia in grado di proporsi credibilmente al governo delle istituzioni nazionali e soprattutto locali. Insomma, la Lega vince al sud non solo grazie alle «gesta» del Capitano, ma anche per le attività dei cavalieri e le iniziative dei fanti che stanno popolando l'esercito del partito al sud.

In questa prospettiva, proprio il risultato delle elezioni europee si propone come strumento per una prima valutazione della formazione al sud di personale politico leghista. Più specificamente, analizzeremo la distribuzione del voto di preferenza per stabilire se la Lega riesca a sollecitare consensi facendo leva non solo sull'appeal di Salvini, ma anche sulla capacità di altri candidati di attrarre consensi personali convogliandoli a favore della lista.

La nostra analisi ovviamente si colloca dentro la discussione più generale che riguarda la trasformazione dei partiti, del loro manifesto, ma soprattutto del loro modello organizzativo. Nella prima parte del contributo saranno evidenziati gli aspetti che in letteratura sono considerati come particolarmente importanti per descrivere l'evoluzione e la trasformazione della forma partito. La seconda parte del saggio è dedicata all'analisi dei risultati alle elezioni europee nelle regioni meridionali e in particolare della distribuzione del voto di preferenza. Essa sarà sviluppata sulla base di una ipotesi che riconosce il merito della performance della Lega all'appeal della 
leadership nazionale per la sua capacità di ri-orientare la proposta politica del partito su temi "nazionali" e che tuttavia, segnala il contributo di alcuni esponenti politici locali al successo del partito di Salvini. Nella terza parte saranno descritte le dinamiche di costruzione del consenso in alcuni specifici "esemplari" contesti per valutare se e in quale misura la crescita elettorale della Lega sia stata supportata dal personale politico meridionale.

\section{Il quadro di riferimento}

I partiti sono esposti al cambiamento. Il mutamento dei partiti si presenta con diversi gradi di intensità perché essi evolvono secondo stadi simili, ma anche in risposta alle specifiche situazioni sociali e politiche in cui competono. Inoltre, i partiti nel loro processo di adattamento sono condizionati dalle circostanze in cui sono nati e si sono sviluppati (Bartolini e Mair 2001, pp. 328-330). Il cosiddetto «modello originario» orienta lo sviluppo successivo di un determinato partito. Combinando le teorie formulate da diversi autori (Duverger 1961, pp. 15-31; Bartolini 1996, 518; Panebianco 1982, pp. 104-110) nel modello originario possiamo far rientrare i seguenti fattori: l'ambiente in cui il partito ha origine (interna/esterna), il modo in cui è iniziata la costruzione dell'organizzazione (diffusione/penetrazione), la presenza o l'assenza di istituzioni esterne, in particolare di associazioni di interessi, che sponsorizzano la nascita del partito, il carattere della leadership (puro o situazionale ossia legato allo stress sociale).

Le dimensioni investite da processi di trasformazioni riguardano l'organizzazione in senso ampio e l'ideologia ossia il manifesto culturale. Le due dimensioni sono strettamente correlate anche se spesso gli studiosi se ne occupano spesso separatamente.

Pur riconoscendo il grande valore degli studi che si dedicano alla ideologia dei partiti e al cambiamento dei tratti ideologici di alcune delle grandi famiglie di partito, la nostra riflessione prescinde da questi aspetti politico-culturali per concentrarsi sugli aspetti organizzativi del partito. Ovviamente il cambiamento dei partiti può essere osservato non solo nel nostro paese, ma anche nelle altre democrazie occidentali e specificamente europee. A partire dal seminale contributo di Kirchheimer (1966) l'evoluzione del partito politico è stata descritta come progressivo allontanamento dal modello del partito di massa. E questo andamento appare adeguato a descrivere la Lega che per la prima parte della sua vita 
politica è stata assimilata a un partito tradizionale fortemente radicato nelle comunità locali (Diamanti 1993).

Molte ricerche hanno studiato l'organizzazione dei partiti individuandone e isolandone le diverse dimensioni. Lo schema analitico forse più noto e spesso adottato nelle ricerche sui singoli partiti fa riferimento a tre facce. Il party on the ground riguarda l'organizzazione di base ossia la membership e le strutture periferiche, il party in central office concerne gli apparati burocratici e il quadro dirigente centrale e nazionale, il party in public office è relativo alla presenza e all'articolazione e alle proiezioni dei partiti nelle istituzioni statali e ai loro reciproci rapporti.

Le analisi condotte secondo questo schema hanno permesso di rilevare tendenze di carattere generale come la crescente egemonia del party in public office e, di conseguenza, la «statalizzazione» dei partiti che tendono a proporsi come organi dello Stato e dall'altra la «partitizzazione» delle istituzioni pubbliche largamente occupate dagli uomini di partito (Panebianco 1982; Calise 1992; Katz e Mair 1994). Ai fini della nostra riflessione l'acquisizione più importante che deriva da queste ricerche riguarda il ruolo dominante degli eletti nell'organizzazione

L'approccio organizzativo ha ispirato altri schemi analitici. Quello più promettente ai fini del nostro ragionamento è strutturato attorno alle funzioni svolte dai partiti e si articola in tre dimensioni: party in Electorate, as Organization e in Government (Key 1964; Dalton e Wattenberg 2000). Il partito as Organizzation seleziona, recluta e forma i gruppi dirigenti, articola e aggrega gli interessi politici; il partito in Government forma le maggioranze parlamentari, organizza il governo ma anche l'opposizione all'Esecutivo, implementa gli obiettivi politici, controlla l'amministrazione, assicura la responsabilità dell'azione di governo, garantisce stabilità e continuità all'azione di governo; il partito in Electorate informa i cittadini e fornisce loro un criterio per semplificare la scelta di voto, genera simboli di identificazione e lealtà, mobilita i cittadini al voto (Dalton e Wattenberg 2000, pp. 5-10).

La nostra attenzione sarà rivolta al partito come organizzazione e in particolare alla selezione dei candidati e al loro rendimento elettorale. Tra l'altro il reclutamento e la formazione del personale politico resta ancora una delle funzioni fondamentali dell'attività di ogni partito (Panebianco 1982; Melchionda 1996).

Sono due gli aspetti specifici che riguardano il cambiamento dei partiti e che possono essere rinvenuti anche nella trasformazione della Lega. Il primo riguarda lo spostamento delle attenzioni della Lega dalla società alle istituzioni. La Lega, insomma, tende ad assomigliare sempre più ad altri 
partiti che progressivamente sono diventati più remoti dalla società (Mair 1992). In passato per descrivere il partito di Bossi a lungo è stato evocato il tradizionale modello del partito di massa. E la Lega aveva fatto del suo insediamento elettorale la bandiera, il manifesto politico e il suo punto di forza (Diamanti 2006). Il successo della Lega al Nord era stato collegato alla sua capacità di essere presente nelle comunità locali di svolgere attività e iniziative dentro quelle comunità, di farsi portatore dei loro interessi e delle loro istanze. Progressivamente questo modello si è indebolito come testimonia il calo significativo di iscritti al partito (Passarelli eTuorto 2018). Corrispondentemente la Legga ha accresciuto la presenza nelle amministrazioni locali. In questo modo non solo è riuscita a supplire al calo degli iscritti e della militanza, ma si è anche dotata di gruppi dirigenti capaci di amministrare e governare. Questa fazione degli eletti ha progressivamente accresciuto la propria influenza anche dentro il partito.

Lo spostamento di enfasi dalla società alle istituzioni si manifesta anche nella modalità di formazione dei gruppi dirigenti e nella crescente importanza dentro la Lega del personale formatasi nelle attività istituzionali, al vertice degli Esecutivi o anche nelle assemblee elettive.

L'indebolimento della leadership di Umberto Bossi si era accompagnato ad un significativo ridimensionamento elettorale del partito. Dopo il breve interregno di Roberto Maroni, la nuova leadership ha provato a far fronte a quella crisi tentando di estendere la propria presenza in area diverse da quelle tradizionali del nord del Paese. E se in un passato anche relativamente recente faceva notizia e scalpore l'insediamento di un esponente della Lega al vertice di un comune delle Marche, negli ultimi anni si possono contare numerosi casi di questo genere. Tuttavia, il successo elettorale della Lega non sembra sia stato accompagnato o preceduto da attività di insediamento sociale e prepolitico del movimento di Bossi.

Negli anni più recenti Salvini ha provato ad accrescere il patrimonio di consensi alla Lega nel Mezzogiorno. Prima ancora che sul piano elettorale, la Lega sembra abbia raccolto molte adesioni da nuovi e vecchi politici che si sono anche resi disponibili ad essere candidati sotto il simbolo della Lega. L'organizzazione della Lega nel Mezzogiorno fa leva soprattutto sulla domanda di protagonismo di questo personale politico. Si tratta di un modello di partito molto lontano da quello che è stato sperimentato al Nord. Ma si tratta di un partito vero o che potrebbe farsi "vero". D'altro canto come avvertiva Duverger «Un partito non è una comunità ma un insieme di comunità, una riunione di piccoli gruppi sparsi per il paese (sezioni, comitati, associazioni locali ecc.) collegati da istituzioni coordinatrici» (Duverger 1961, p. 53). Il punto tuttavia è che al sud il partito nasce secondo 
modalità diverse da quelle che ne hanno favorito il consolidamento al nord, anche se tra alti e bassi, tra successi e rovesci elettorali. Si tratta di un modello che assume il ruolo delle personalità individuali come principale strumento dell'azione politica e come risorsa elettorale. Ovviamente, la personalizzazione riguarda la leadership nazionale che, al di là delle caratteristiche individuali di Salvini, è riuscita ad accentrare nelle proprie mani la comunicazione, la strategia politica generale. Ma l'affidamento della struttura organizzativa locale a personalità individuali più $\mathrm{o}$ meno insediate localmente ha lo stesso segno di personalizzazione dell'offerta.

Accanto a quella che è stata definita la personalizzazione accentrata e che deriva dalla debordante presenza di Salvini, la Lega sembra valorizzare anche la personalizzazione decentrata che riguarda ruoli e funzioni riferibili non ai vertici degli Esecutivi o del partito, ma a ruoli e funzioni che riguardano le assemblee elettive nazionali e anche locali (Balmas, M., Rhat, G., Sheafer, T., e Shenhav, S. R., 2014).

\section{Elezioni europee: l'inusitata crescita dei consensi per la Lega al Sud}

Le Europee del maggio 2019 hanno riconosciuto La Lega di Salvini come partito nazionale, almeno sul terreno elettorale. Permangono gli squilibri territoriali nei risultati della Lega; al sord è saldamente il primo partito, avendo ottenuto più del $40 \%$ di voti (Tabella 1 ). Tuttavia, nelle regioni meridionali ha compiuto un significativo passo in avanti avendo superato abbondantemente un quinto dei consensi sia nella circoscrizione meridionale $(23,5 \%)$ che in quella insulare $(22,4 \%)$ E soprattutto ha sbaragliato la concorrenza di Forza Italia e ha ormai una consistenza tale da risultare seconda solo al M5S.

TAB. 1 - Elezioni Europee 2019 - Voti (\%) alla Lega per circoscrizione elettorale.

\begin{tabular}{ll}
\hline Circoscrizione & Lega \\
\hline Nord-Occidentale & 40,7 \\
Nord-Orientale & 41,0 \\
Centrale & 33,5 \\
Meridionale & 23,5 \\
Insulare & 22,4 \\
\hline Italia & 34,3 \\
\hline
\end{tabular}

Fonte: nostra elaborazione su dati del ministero dell'Interno. 
Rispetto alle elezioni politiche dell'anno prima, la Lega Salvini Premier nelle regioni del sud (le due circoscrizioni Meridionale e Insulare) ha conquistato più di 20 punti percentuali in più. Dunque, rispetto al 2018 la platea degli elettori della Lega si è notevolmente ampliata. Ovviamente, la percentuale dei consensi alla Lega presenta differenze fra le otto regioni meridionali ben più significative rispetto alle passate elezioni. In Abruzzo, che nel 2018 aveva tributato il 13,9\% dei consensi al partito di Salvini, la Lega è al 35,3\%, confermandosi la regione meridionale più verde. In Sardegna la Lega è passata dal 10,8\% della Camera nel 2018 al 27,6\% del 2019. La Puglia è fra le regioni meridionali quella che fa registrare il maggiore incremento di consensi alla Lega dal 2018 al 2019, passando dal $6,2 \%$ della Camera al 25,3\% delle Europee. Seguono in questa graduatoria della Lega al sud, Molise con il 24,3\% e Basilicata con il 23,3\%. Nelle altre regioni (Campania, Calabria e Sicilia) la Lega Salvini Premier si attesta intorno al 20\%, probabilmente per la maggiore tenuta e presenza del M5S.

\section{I voti per la Lega al Sud: dalle elezioni politiche alle europee passando per le elezioni regionali}

La «conquista» elettorale delle regioni meridionali del partito di Salvini, iniziata con le politiche del marzo 2018, ha avuto una straordinaria progressione con le europee del maggio 2019. I numeri certificano l'avanzata leghista nel sud in maniera inequivocabile. Nelle elezioni europee del 2014, la Lega Nord nelle due circoscrizioni del sud poteva registrare solo una presenza simbolica con lo $0,75 \%$ nella circoscrizione meridionale e lo $0,99 \%$ nella circoscrizione insulare. Nelle elezioni politiche dell'anno prima, la Lega Nord alla Camera nelle otto regioni meridionali, pur presentando liste di candidati quasi esclusivamente del territorio, aveva ottenuto risultati molto più prossimi allo zero che all'uno percento: Abruzzo e Molise 0,18\%, Campania 0,29\%, Puglia 0,07\%, Basilicata 0,12\%, Calabria $0,25 \%$, Sicilia $0,19 \%$ e Sardegna $0,19 \%$. Possiamo, perciò, affermare che, fino al 2014, la Lega Nord era un partito territoriale in un rapporto di vicendevole disinteresse con gli elettori delle regioni del Sud. La competizione nella coalizione di centrodestra per la conquista della leadership e del governo del Paese contribuisce, senza ombra di dubbio, al cambiamento culturale, all'inversione, nel rapporto Lega e regioni meridionali. Salvini ha ben presente nel 2018 che se vuole primeggiare nella coalizione, sopravanzando Forza Italia, deve erodere consensi alla 
formazione di Berlusconi nelle aree in cui questa era, fin a quel momento, più forte (Diamanti 2003; 2006) ovvero in alcune regioni meridionali.

Le elezioni politiche del 4 marzo 2018 hanno segnato questo passaggio da Lega Nord alla Lega Salvini Premier. Nelle otto regioni meridionali, pur in misura disomogenea, la Lega di Salvini ha ottenuto il $6,3 \%$ dei voti validi (Tabella 2 ). Si tratta di un risultato ben al di sotto di quello del $17,4 \%$ ottenuto con riferimento all'intero territorio nazionale. Tuttavia, esso rappresenta un significativo avanzamento per un partito che prima di allora era inesistente, per presenza, opinione ed organizzazione, nei territori delle regioni meridionali. Il successo della Lega al sud fa il paio con la straordinaria affermazione del Movimento 5 Stelle. Le percentuali di voti ottenute dalle due liste nelle otto regioni meridionali distano un abisso (Lega 6,3\%, M5S 46,1\%) ma entrambe hanno un significato importante se consideriamo le condizioni di partenza dei due concorrenti. Il M5S è entrato nel parlamento la prima volta nel 2013 con uno straordinario consenso, e nel 2018 è risultato nettamente il primo partito in Italia aumentando di oltre sette punti percentuali i suoi voti, grazie soprattutto agli elettori del Sud. Per la Lega al sud, invece, il 2018 rappresenta l'anno zero, un'elezione per un partito che prima di allora era stato completamente assente nelle regioni meridionali e che giocoforza - per arrivare al governo del Paese - ha dovuto accantonare o trasformare i mantra retorici "Roma ladrona" e "Prima la Padania" e, nello stesso tempo, reclutare candidati e militanti del territorio in grado di rendere credibile ed organizzativamente sostenibile una tale pretesa politica. Il primo impatto della Lega "nazionale" nelle regioni meridionali, guardando al risultato elettorale, ha fornito le coordinate del nuovo corso che, puntualmente, hanno indicato che la via intrapresa era quella vincente, prima nelle consultazioni delle regioni dove si sono rinnovati consigli e presidenti e, successivamente, nelle Europee del 2019. 
Tab. 2 - Elezioni Camera 2018 e Europee 2019. Consensi a Lega e M5S nelle regioni meridionali.

\begin{tabular}{lllllll}
\hline & \multicolumn{3}{c}{ Camera 2018 } & \multicolumn{4}{c}{ Europee 2019 } \\
\hline Regioni & Lega & M5S & Lega+M5S & Lega & M5S & Lega+M5S \\
\hline Abruzzo & 13,9 & 39,8 & 53,7 & 35,3 & 22,4 & 57,7 \\
Molise & 8,7 & 44,8 & 53,5 & 24,3 & 28,8 & 53,1 \\
Campania & 4,3 & 49,4 & 53,7 & 19,2 & 33,8 & 53,0 \\
Puglia & 6,2 & 44,9 & 51,1 & 25,3 & 26,3 & 51,6 \\
Basilicata & 6,3 & 44,4 & 50,7 & 23,3 & 29,7 & 53,0 \\
Calabria & 5,6 & 43,4 & 49,0 & 22,6 & 26,7 & 49,3 \\
Sicilia & 5,1 & 48,7 & 53,8 & 20,8 & 31,2 & 52,0 \\
Sardegna & 10,8 & 42,5 & 53,3 & 27,6 & 25,7 & 53,3 \\
\hline Valore & & & \multicolumn{5}{c}{} \\
mediano & 6,3 & 44,6 & 53,4 & 23,8 & 27,8 & 53,0 \\
\hline Fonte:
\end{tabular}

Fonte: nostra elaborazione su dati del ministero dell'Interno.

Nelle elezioni per la Camera del marzo 2018 il dato della Lega è abbastanza omogeneo nelle nostre otto regioni meridionali. Le percentuali più elevate si registrano in Abruzzo e Sardegna. L'Abruzzo è la regione meridionale più prossima alle regioni centrali dove già da tempo la Lega aveva ottenuto un elevato numero di consensi. In Sardegna, probabilmente, alla Lega sono arrivati anche i consensi di formazioni autonomiste dell' isola.

Se si sommano le percentuali di voto al Movimento 5 Stelle e alla Lega alle elezioni del 2018, si può cogliere una coincidenza che ci suggerisce un'osservazione: in cinque regioni la somma di queste due liste è intorno al 53\% e nelle restanti tre è intorno al 50\%. Questa coincidenza, letta da un'altra prospettiva, suggerisce che quando la Lega è più forte elettoralmente, i voti vengono sottratti al M5S. Ed ancora, che le due liste sembrano essere l'una il complemento dell'altra. Lega e M5S sono stati, da più parti, definiti come partiti populisti o, quantomeno, due soggetti che fanno leva su retoriche e appelli populisti.

Le regioni nelle quali la Lega ha ottenuto migliori risultati alle elezioni europee sono quelle che hanno rinnovato fra le due elezioni, politiche ed Europee, i rispettivi consigli e dove la Lega ha ottenuto i maggiori consensi eleggendo, conseguentemente, un buon numero di rappresentanti nel consiglio regionale. La presenza di eletti nelle istituzioni locali, oltre che condizione necessaria, è sicuramente un buon indicatore del radicamento del partito sul territorio. Sono, soprattutto, gli eletti ai diversi livelli, comunale e regionale, che 
si mobilitano in ogni elezione stabilendo collegamenti con l'elettorato con una ricaduta positiva sui livelli di consenso al partito. In Puglia dove non è stato ancora rinnovato il consiglio regionale, il successo può essere collegato alla presenza nella lista circoscrizionale di candidati conosciuti sul territorio i quali hanno contribuito con i voti di preferenza personali a far lievitare il risultato del partito in quella regione ${ }^{1}$.

La crescita elettorale della Lega nelle regioni meridionali si può valutare meglio osservando come sono cambiate le percentuali aggregate del voto in queste regioni rispetto al dato nazionale della Lega. Nelle Europee del 2014 le regioni meridionali avevano contribuito al risultato nazionale della Lega del solo 3,9\%. Alle elezioni per la Camera del 2018 il complessivo voto alla Lega nel meridione rappresentava 1'11,7\% del totale e alle ultime Europee si è saliti al 19,0\%. Anche se l'incremento elettorale nel meridione è stato eccezionale, per la Lega permane «una frattura territoriale» poiché «i maggiori azionisti, le principali aree di forza e di insediamento [sono] nel Nord» (Passarelli e Tuorto 2018).

I sondaggi dopo le elezioni del 2018 accreditavano l'ipotesi che la Lega stesse diventando sempre più un partito capace di raccogliere quote elevate di consensi su tutto il territorio nazionale. L'avanzata della Lega nelle regioni del Sud è stata attestata nelle elezioni regionali di Molise, Abruzzo, Sardegna e Basilicata. In queste quattro regioni, l'apporto della Lega, quasi sempre, è stato determinante per la vittoria della coalizione di centrodestra. In Molise, nelle regionali svoltesi il 22 aprile 2018, un mese e mezzo dopo le politiche, la Lega ha praticamente confermato i voti delle politiche $(8,2 \%$ alle regionali, $8,7 \%$ alla Camera). Ma occorre considerare che alle regionali sono presenti diverse liste non partitiche che sottraggono voti alle liste ufficiali grazie a candidati che intercettano molti consensi personali.

In Abruzzo, alle regionali svoltesi il 10 febbraio 2019, la Lega è diventata il primo partito con il $27,5 \%$, cioè 13,6 punti in più, il doppio, della percentuale che era stata ottenuta l'anno prima alle politiche. La seconda lista è il M5S con il $19,7 \%$, terzo il PD con $11,1 \%$ e quarto FI con il $9,0 \%$. Anche in Abruzzo si è verificato il ridimensionamento dei partiti nazionali quale conseguenza della presenza, nelle due più importanti coalizioni, di liste non partitiche (De Luca 2018) e formate all'occorrenza,

\footnotetext{
1 Massimo Casanova, assiduo frequentatore della Puglia, imprenditore proprietario del Papeete, locale sulla riviera romagnola da dove Salvini ha condotto un'azione mediatica nella politicamente caldissima estate del 2019, ha ottenuto circa 38.000 voti di preferenza in Puglia. Ma erano presenti altri importanti candidati pugliesi quali Andrea Caroppo con circa 41.000 voti di preferenza.
} 
anche con candidati politici di lungo corso, con il fine di contribuire alla vittoria del candidato presidente della coalizione.

Nello stesso mese di marzo 2019 si sono svolte le elezioni regionali in Sardegna con la vittoria del candidato della coalizione di centrodestra che comprendeva diverse liste non partitiche e regionali. La Lega Salvini Sardegna è risultata il primo partito della coalizione con 1'11,4\%. Il presidente eletto, Christian Solinas, già segretario dello storico Partito Sardo d'Azione, era deputato eletto nelle fila della Lega, in seguito al gemellaggio che nelle elezioni politiche del 2018 era avvenuto fra Lega e Partito Sardo d'Azione. La scelta del candidato presidente evidenzia il crescente peso politico della Lega di Salvini all'interno della coalizione di centrodestra nelle regioni del Sud dove, in precedenza, Forza Italia dettava regole e programmi e designava i candidati ai vertici esecutivi. Il risultato ottenuto dalla Lega di Salvini, pur essendo superiore di solo 1,4 punti percentuali rispetto alla Camera 2018, è significativo se consideriamo che le liste provinciali dei candidati consiglieri della Lega non avevano grandi intercettori di voti di preferenza, a differenza di altre liste. Si potrebbe desumere che il voto alla Lega Salvini Sardegna si basa essenzialmente sull'opinione degli elettori del partito di Salvini, un partito che pur carente di organizzazione nella regione «ha un suo brand riconoscibile e un leader indiscusso nell'arena nazionale» (Passarelli e Tuorto 2018).

L'indice di preferenza risulta elevato a conferma del criterio di scelta utilizzato dagli elettori del voto alla persona, anziché al partito. E il voto di preferenza viene assegnato dall'elettore soprattutto a candidati del territorio. Questo sta a significare che la Lega in queste elezioni regionali ha saputo interpretare strategicamente il meccanismo elettorale, riuscendo a reclutare candidati noti sul territorio, in grado di conquistare consensi personali. Cioè ai voti che possiamo definire di «opinione» in favore di Salvini e della Lega, si sono aggiunti i consensi ottenuti dai candidati consiglieri, espressione del territorio. In una fase politica contrassegnata dalla estrema volatilità elettorale, questi candidati rappresentano un punto importante per stabilire il radicamento di un partito sul territorio, radicamento che può significare affermazione e continuità nel tempo del partito.

Due mesi prima delle Europee, la regione Basilicata ha eletto il nuovo presidente e il consiglio regionale. Dal 1995, il presidente della giunta è stato un esponente del centrosinistra. La coalizione di centrodestra, grazie all'apporto della Lega Salvini Basilicata, ha vinto nettamente sul centrosinistra e sul M5S che poteva contare su un $44,4 \%$ ottenuto alle politiche di un anno prima. La Lega è il secondo partito in Basilicata e nettamente il primo partito della coalizione. In Basilicata, così come abbiamo visto per la Sardegna, le liste di Lega Salvini Basilicata non riescono ad avere candidati campioni delle 
preferenze che contribuiscono al successo della lista. Contrariamente a quanto solitamente accade, cioè le liste che hanno più candidati che si contendono il voto di preferenza ottengono risultati migliori, in Basilicata le due liste che hanno avuto il più basso indice di preferenza hanno ottenuto la più alta percentuale di voti: M5S (indice di preferenza 34,9\%) e Lega Salvini Basilicata (indice di preferenza 39,0\%).

Una campagna elettorale con la presenza diretta del ministro Salvini in Basilicata in tantissimi comuni della regione hanno prodotto per la Lega lo straordinario risultato di quasi 13 punti percentuali in più rispetto alle politiche dell'anno prima. Una prova sul campo della Lega "partito nazionale" e viatico dell'imminente campagna elettorale per le Europee.

\section{Il voto di preferenza}

L'analisi fin qui sviluppata ha evidenziato che la Lega alle elezioni europee ha raggiunto nelle regioni meridionali un elevato livello di consensi, avendo superato il $20 \%$ dei voti. Quel successo può essere attribuito all'appeal personale di Salvini e alla efficace campagna mediatica che è stata sviluppata dal segretario della Lega e abilmente orchestrata dai suoi collaboratori e consulenti. In realtà, Salvini sin dal suo insediamento alla guida della Lega aveva avviato una strategia di ri-orientamento della proposta politica del partito ottenendo risultati positivi già alle elezioni politiche del 2018. E, in effetti, la comunicazione si era focalizzata su temi aventi un orizzonte nazionale e non più solo regionale nel tentativo di intercettare sensibilità, preferenze e bisogni dei cittadini dell' intero paese, anche delle regioni meridionali.

Salvini, che nel frattempo aveva assunto anche la carica di Ministro dell'Interno e Vice Presidente del Consiglio dei ministri, ha sviluppato una insistente campagna mediatica sulla questione della regolazione dei flussi migratori intrecciandola al problema della sicurezza. Le politiche promesse o realizzate di contrasto all'immigrazione, i decreti sicurezza, i provvedimenti di revisione dell'istituto della legittima difesa hanno rassicurato e blandito molti elettori meridionali. In queste aree i cittadini si sentono più esposti da un lato ai rigori della crisi economica e percepiscono i migranti come competitori e concorrenti sleali e dall'altro avvertono l'esigenza di una maggiore sicurezza di fronte alla diffusione della criminalità attribuita all'arrivo dei migranti. Insomma, il securitarismo ha evidentemente fatto breccia nel Mezzogiorno del Paese procurando consensi elettorali a Salvini che, anche grazie alla sua veste di Ministro dell'Interno, ne è diventato il più credibile alfiere. 
Questa interpretazione dei risultati elettorali è ricorrente e prevalente tra gli osservatori ed è, a nostro avviso, plausibile e condivisibile. D'altro canto già alle precedenti elezioni politiche il M5S aveva dimostrato che una buona parte dell'elettorato meridionale è disponibile a modificare la propria scelta di voto a favore di forze politiche che si mostrano sensibili su questioni percepite come urgenti e sono disponibili a farsi carico di domande e istanze cruciali. Nel 2018 il tema fondamentale era il sostegno di fronte alla crisi economica, alle elezioni europee la preoccupazione era diventata soprattutto la sicurezza. Tuttavia, la nostra domanda riguarda non tanto le ragioni dell'ampiezza del consenso della Lega al sud, ma anche la profondità di questo inatteso consenso o più precisamente il potenziale di stabile insediamento della Lega al sud. Un insediamento basato non solo su fattori contingenti come l'appeal di un leader, ma su elementi organizzativi che valicano la durata di una leadership. Insomma, ci proponiamo di sviluppare una prima analisi sulla capacità della Lega di consolidare i consensi nel Mezzogiorno attraverso la diffusione di un tessuto organizzativo, seppure diverso da quello sperimentato nelle regioni settentrionali nella prima fase della vicenda politica del partito di Bossi.

La Lega aveva sviluppato la propria iniziativa nelle regioni settentrionali enfatizzando la forte identificazione con quelle aree anzi utilizzando il territorio contro la politica (Diamanti 2003), ossia contro il centro della politica. Le modalità, e lo spirito con cui la Lega si era insediata nelle regioni settentrionali erano state specifiche e particolari e per questo non replicabili. Tra l'altro, anche quella struttura mostra chiari segnali di cambiamento. È stato infatti evidenziato e documentato che il numero degli iscritti alla Lega è diminuito e che anche le strutture di base hanno ridotto le loro attività (Passarelli e Tuorto 2018). La Lega sembra vivere una parabola analoga a quella dei tradizionali partiti. L'attivismo dei militanti è stato affiancato dall'iniziativa degli eletti i quali garantiscono una continuità di iniziative e di attività e, al contempo, dedicandosi a tempo pieno alla politica hanno accresciuto il peso e l'influenza della direzione del partito. In questo modo il professionismo occulto (Verzichelli 2010) è diventato sempre più importante nella vita della Lega. In generale il baricentro delle attenzioni della Lega si è spostato dalla società alle istituzioni e gli eletti hanno accresciuto la loro importanza se non direttamente nel processo decisionale sicuramente nella vicenda elettorale.

In capo ai candidati ricade spesso la conduzione della campagna elettorale e la responsabilità del successo o del fallimento. E ciò accade per $\mathrm{i}$ candidati ai vertici esecutivi, ma anche quelli che si propongono alle assemblee elettive. Analizzare il rendimento elettorale dei candidati risulta importante da due punti di vista. In primo luogo segnala la capacità della Lega di dotarsi di 
una struttura organizzativa almeno con riferimento allo specifico aspetto del reclutamento del personale politico. In secondo luogo si può valutare l'apporto dei candidati alla performance elettorale della Lega.

In sintesi noi ipotizziamo che il successo della Lega al Sud sia in qualche in qualche misura riferibile anche alle strategie organizzative della Lega e in particolare al reclutamento di personale politico autoctono capace di proporsi efficacemente nella competizione per la elezione dei rappresentanti al Parlamento europeo.

Possiamo valutare la fondatezza di questa ipotesi osservando la distribuzione del voto di preferenza. In questa prospettiva focalizzeremo la nostra analisi su alcune delle regioni incluse nelle due circoscrizioni Sud e Isole. In particolare, l'analisi adotta la nuova classificazione proposta dall'Istituto Cattaneo che divide la precedente area meridionale in due zone: Sud (Campania, Molise, Puglia, Basilicata Calabria e Sicilia) e Centro (Lazio, Abruzzo e Sardegna). Questa nuova classificazione, rispetto a quella precedente, è stata elaborata con l'intento di individuare una zona geopolitica Sud più omogena in ordine ai tassi di partecipazione al voto $\mathrm{e}$, più in generale, al comportamento di voto inclusa la propensione ad esercitare il diritto e la facoltà di esprimere una preferenza.

In secondo luogo, poiché questa nuova classificazione è stata progressivamente utilizzata in molti studi elettorali, il lettore potrà valutare $\mathrm{i}$ tassi di preferenza alle europee riferendole ad altri tipi di consultazione e alle analisi proposte per precedenti elezioni europee. In altre ricerche è emersa una maggiore propensione ad utilizzare il voto di preferenza nelle regioni meridionali individuate in base alla configurazione adottata in questo paragrafo (Fruncillo 2006, 2016).

Preliminarmente è utile riportare i risultati nelle regioni che qui abbiamo considerato Sud (Molise, Campania, Puglia, Basilicata, Puglia e Sicilia), confrontandoli con quelli riferiti alle altre aree geopolitiche del paese (Tabella 3).

In primo luogo è opportuno segnalare che, come era nelle nostre attese, il Sud si differenzia dalle altre zone per quanto riguarda la percentuale dei votanti che si attesta al $44,9 \%$ ben cinque punti in meno del livello di partecipazione calcolato per le regioni incluse nel Centro (Abruzzo, Lazio e Sardegna) e più di 16 punti percentuali al di sotto della quota di partecipanti rilevata per le regioni della zona rossa. Tra le regioni del Sud solo in Puglia e in Molise si registra un numero di votanti paragonabile a quello osservato nelle altre zone. E solo in Molise la percentuale di votanti è superiore a quella del Centro. 


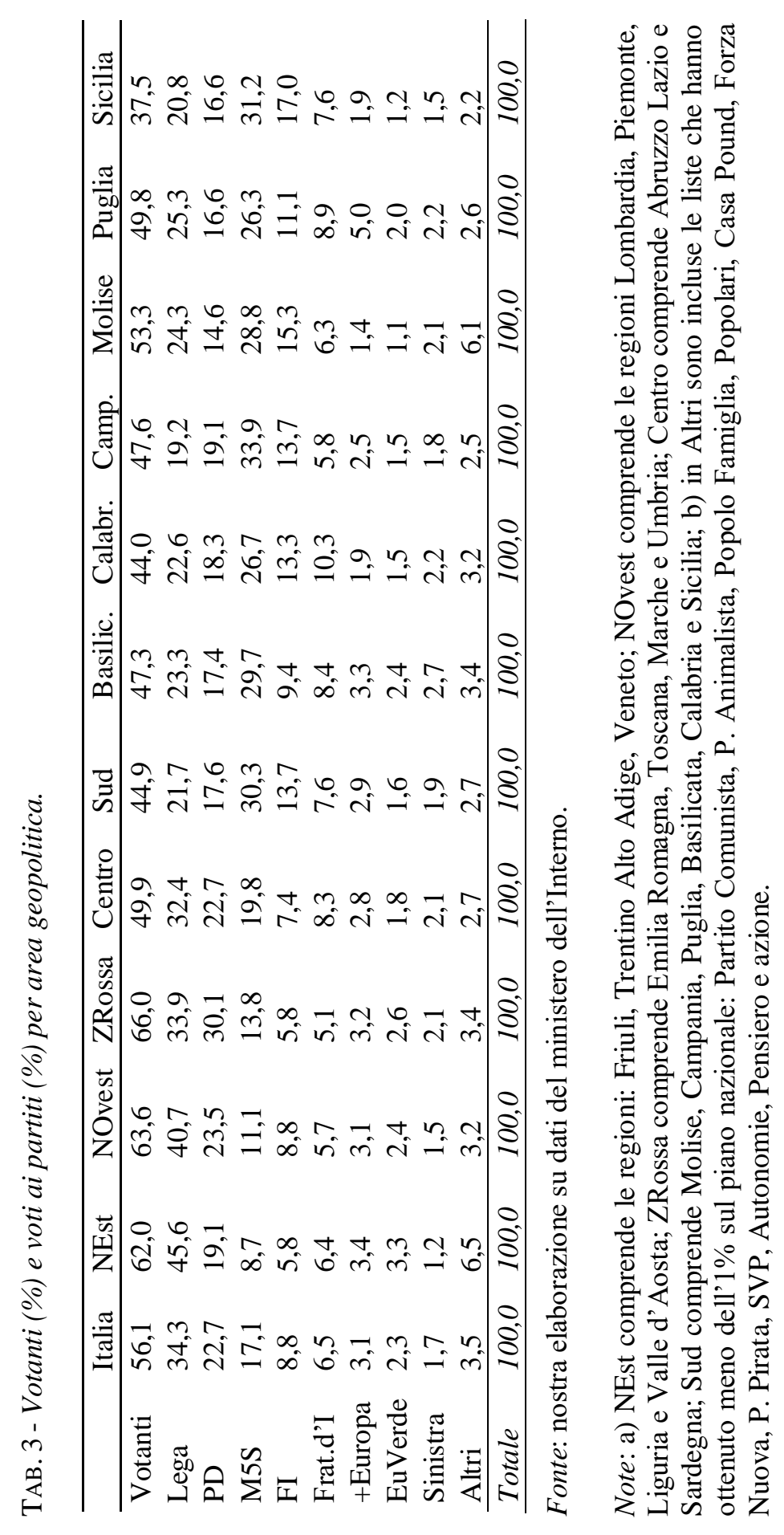


Anche per quanto riguarda la distribuzione dei consensi ai partiti nelle regioni del Sud si rilevano risultati piuttosto diversi rispetto a quelli osservati nelle altre aree geopolitiche e persino rispetto alle regioni del Centro. Sono soprattutto i risultati del M5S e della Lega a segnare la specificità del Sud. Infatti, il M5S ottiene le migliori performance al sud, mentre la Lega in queste regioni paga dazio seppure in misura assai meno rilevante rispetto al passato. Ad ogni modo, il confronto tra i partiti nel sud si è risolto a favore del M5S che ha ottenuto il 30,3\% dei consensi, dieci punti in più rispetto ai consensi raccolti nelle regioni del Centro $(19,8 \%)$ e più di venti rispetto a quelle del Nord Est (8,7\%). Nonostante il considerevole calo di consensi rispetto alle politiche dell'anno precedente $(47,3 \%)$ le regioni del Sud, e in particolare la Campania la Sicilia, restano la roccaforte del partito di Di Maio. Oltre al M5S anche Forza Italia, Fratelli d'Italia e La sinistra raccolgono al sud una percentuale di voti superiore a quella ottenuta sul piano nazionale.

Al contrario al sud restano al di sotto della quota di voti ottenuta a livello nazionale il PD, + Europa, i Verdi e la Lega. Tuttavia, occorre segnalare che, rispetto al 2018, mentre FI perde 7 punti essendo passata dal $20,7 \%$ al $13,7 \%$ e il M5S ne lascia addirittura 17 avendo ottenuto il 30,3\% a fronte del 47,3\% conseguito nel 2018, la Lega, al contrario, ha guadagnato, rispetto al 5,3\% ottenuto nel 2018, più di 16 punti percentuali (Fruncillo 2018). Ci siamo quindi chiesto se il risultato possa essere attribuito ad elementi contingenti legati alla persona di Salvini, al suo appeal, alla sua capacità di stare nel dibattito e di utilizzare con efficacia i mezzi di comunicazione vecchi e nuovi o se esso possa essere in qualche misura attribuito anche ai candidati in lista che potrebbero essere considerati la struttura organizzativa essenziale della Lega al sud su cui sviluppare una strategia di insediamento duraturo. A tale scopo abbiamo analizzato la distribuzione del voto di preferenza nelle regioni meridionali (Tabella 4). 


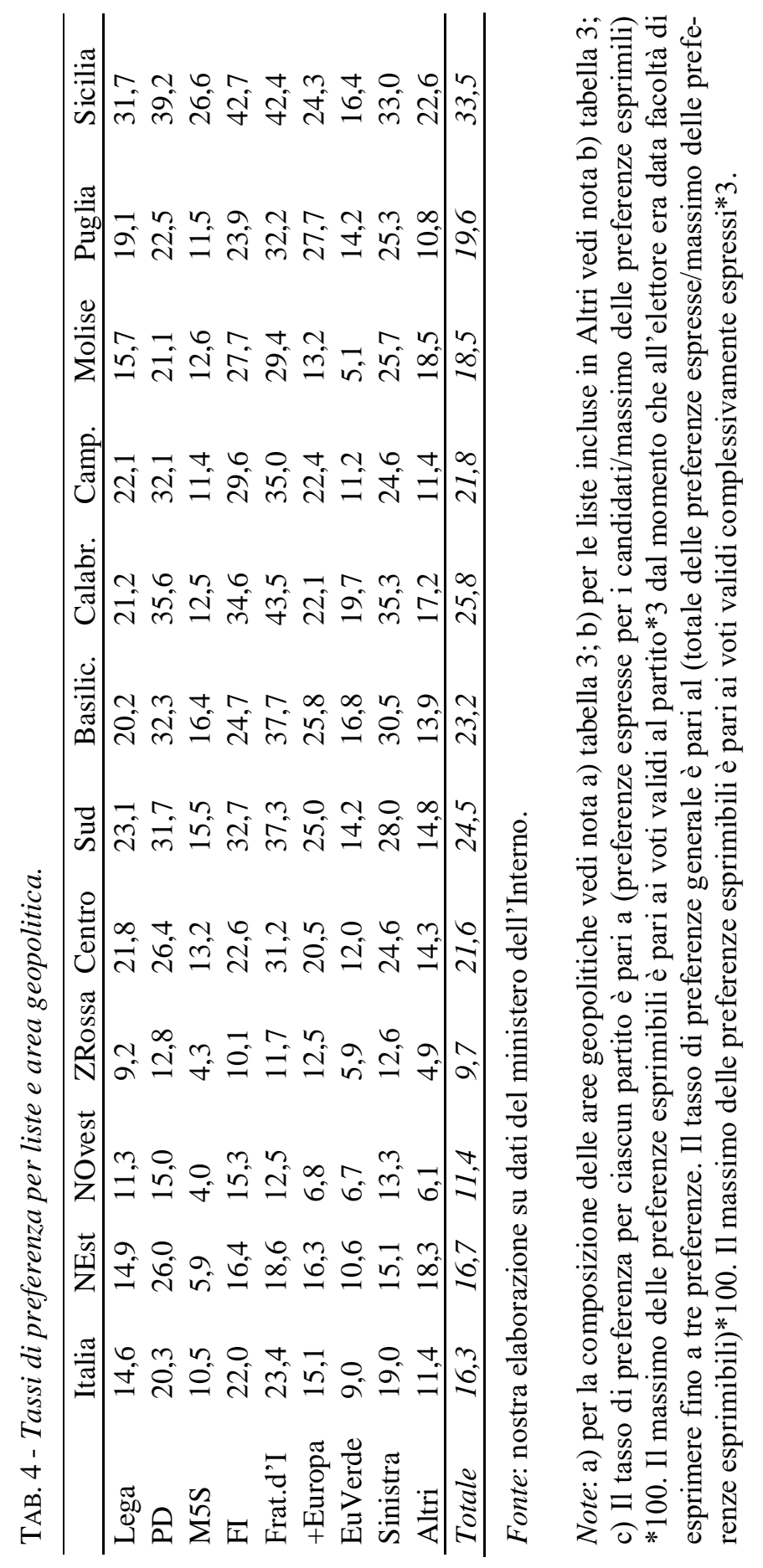


In primo luogo occorre osservare che si rileva un maggiore uso delle preferenze nelle regioni del Sud $((24,5)$ rispetto a tutte le altre aree anche al Centro $(21,6)$. In secondo luogo il tasso di preferenze generale ossia calcolato con riferimento a tutti i partiti e per l'intero Paese è pari a 16,3. La Lega è tra i partiti per cui è stato calcolato un tasso di preferenze a livello nazionale più basso $(14,6 \%)$. Tra i partiti maggiori la Lega, dopo il M5S, è quella per cui vengono espresse meno preferenze. Persino gli elettori di Forza Italia che in passato avevano mostrato scarsa propensione ad utilizzare questo strumento (Fruncillo 2006) sono risultati più generosi di preferenze.

Tuttavia è necessario rilevare che, come accade per altri partiti, la Lega al sud riceve un numero di preferenze assai più consistente e infatti si registra un tasso di preferenze pari a 23,1. Questa tendenza potrebbe essere attribuita ad una certa propensione dei cittadini meridionali ad esprimere un voto al candidato, tuttavia più correttamente il maggior uso delle preferenze dovrebbe essere attribuito all'attivismo dei candidati che sollecitano il voto personale di preferenza.

Dunque è possibile che il voto di preferenza sia indicatore della capacità di mobilitazione dei candidati locali che dunque hanno contribuito ad innalzare il livello dei consensi alla Lega.

Per valutare questo apporto al netto del contributo di Salvini abbiamo calcolato il numero di comuni in cui è il candidato con il maggior numero di preferenze, nell'ipotesi che laddove il candidato più votato è diverso dal capolista, il maggior contributo all'aumento dei consensi elettorali possa essere attribuito a candidati locali (Tabella 5). 


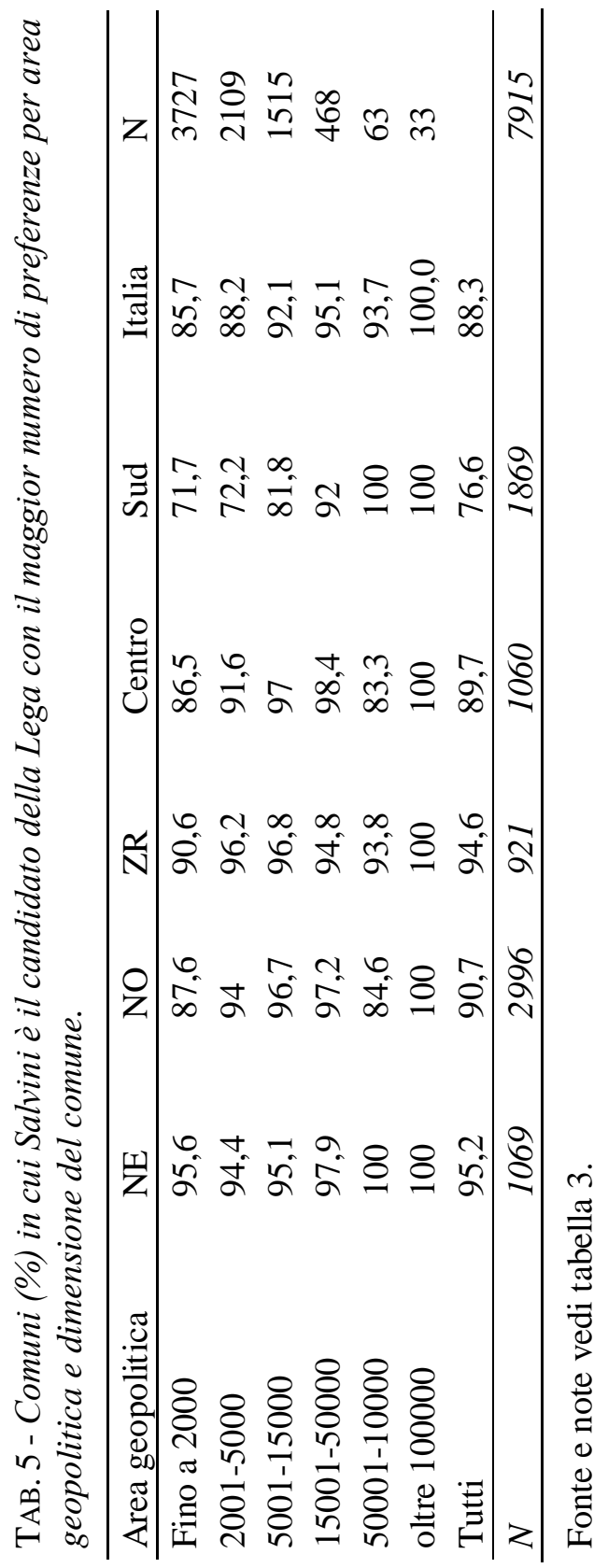


Ebbene, la percentuale di comuni in cui Salvini è il più votato al sud è significativamente più bassa $(76,6 \%)$ di quella calcolata per le altre aree del Paese e sul piano nazionale. Essa peraltro cala ulteriormente per i piccoli comuni con un numero di elettori pari a 5000. In questi casi Salvini è il più votato solo in circa il $72 \%$ dei comuni. Essa invece cresce rapidamente nei comuni più popolosi. Questo dato sembra suggerire che nelle piccole comunità il consenso personale a candidati del territorio o, comunque, sostenuti da politici locali è risultato più importante che altrove per il successo della Lega. In questi comuni spesso le relazioni clientelari sono strutturate da notabili locali piuttosto che da strutture partitiche.

A considerazioni analoghe si perviene se consideriamo il tasso di leadership interna che è pari la rapporto tra il numero di preferenze attribuite a Salvini e il totale delle preferenze raccolte da tutti i candidati della Lega. La nostra ipotesi è che il peso del leader, rispetto a quello degli altri candidati in lista, è maggiore se è più elevata la sua quota di preferenze. Ebbene, il valore di tale indice è di gran lunga superiore al $50 \%$ in tutte le altre aree, mentre si ferma al 47,9 nelle regioni del Sud a conferma della circostanza che in queste aree il peso degli altri candidati è più elevato che altrove.

ТАВ 6 - Indice di leadership (media degli indici calcolati per comune) per area geografica e dimensione comuni.

\begin{tabular}{|c|c|c|c|c|c|c|c|c|}
\hline & & $\begin{array}{l}\text { Fino } \\
\mathrm{a} \\
2000\end{array}$ & $\begin{array}{l}2001- \\
5000\end{array}$ & $\begin{array}{l}5001- \\
15000\end{array}$ & $\begin{array}{l}15001- \\
50000\end{array}$ & $\begin{array}{l}50001- \\
100000\end{array}$ & $\begin{array}{l}\text { Oltre } \\
100000\end{array}$ & Totale \\
\hline \multirow[t]{6}{*}{$\begin{array}{l}\text { Leadership } \\
\text { interna }\end{array}$} & NE & 63.6 & 61,0 & 58.2 & 60.2 & 59,6 & 65.5 & 61,3 \\
\hline & $\begin{array}{l}\mathrm{NO} \\
\mathrm{Z} .\end{array}$ & 63,9 & 66,1 & 65,6 & 68,2 & 62,8 & 68,8 & 64,8 \\
\hline & rossa & 65,0 & 69,9 & 71,7 & 67,3 & 70,1 & 71,9 & 68,8 \\
\hline & Centro & 56,5 & 56,1 & 57,0 & 57,8 & 56,8 & 56,4 & 56,5 \\
\hline & Sud & 46,9 & 45,5 & 49,7 & 53,6 & 56,0 & 59,6 & 47,9 \\
\hline & Totale & 59,7 & 59,1 & 60,1 & 60,4 & 61,3 & 65,0 & 59,7 \\
\hline
\end{tabular}

Fonte e note vedi tabelle 3 e 4; l'indice di leadership interna è calcolato come rapporto tra i voti di preferenza a Salvini sul totale delle preferenze espresse per i candidati della Lega in ciascun comune.

L'analisi induce a proporre alcune osservazioni che sono soprattutto ipotesi di ricerca. Il successo della Lega sembra essere il frutto delle due 
personalizzazioni di cui si è parlato. Il ruolo dei politici locali non è stato ininfluente e suggerisce che la Lega sta provando a radicarsi nel Mezzogiorno a prescindere dall'appeal del leader. In particolare, deve essere valutata con molta attenzione il reclutamento di personaggi politici locali in grado di dare credibilità politica alla proposta della Lega e capaci di sollecitare consensi personali soprattutto in alcune comunità e in alcuni ambienti. Se così fosse il successo della Lega potrebbe consolidarsi in futuro.

Tuttavia occorre considerare che questo personale politico non viene dal nulla, spesso si tratta di personaggi orfani di Forza Italia e del PD, indifferenti ai contenuti della proposta politica, che sono alla ricerca di una loro conferma nel sottogoverno o che ambiscono ad assumere ruoli di direzione nelle amministrazioni locali e regionali. Essi spesso sono mediatori tra centro e periferia. Ciò significa anche che essi sono filogovernativi. La estromissione temporanea della Lega dal governo potrebbe scoraggiare qualcuno, ma potrebbe al contrario rafforzare la loro scelta poiché, in adesione al meccanismo del cane bastonato, potrebbero correre in soccorso del leader leghista.

\section{Il contributo dei candidati al radicamento della Lega nelle regioni meridionali: alcuni casi esemplari}

Un indicatore dell'insediamento e radicamento di un partito, al di là del risultato elettorale, si può riscontrare nella presenza di candidati del territorio, in ogni tipo di elezione, in grado di intercettare consistenti consensi. Se un tempo, nella "Prima repubblica", il partito veniva scelto prima del candidato, ora il criterio che spesso prevale nella scelta dell'elettore è del candidato prima del partito. Per cui l'apporto personale dei candidati al successo di una lista può risultare rilevante. Abbiamo già osservato che in alcune elezioni regionali (Basilicata e Sardegna) il risultato della Lega sarebbe potuto essere ancora migliore con la presenza nelle liste di candidati noti del territorio, attribuendo a questa debolezza del partito il significato di un limitato radicamento territoriale. Nelle elezioni europee la vastità della circoscrizione, spesso, frappone un'oggettiva distanza fra candidati e cittadini, con la conseguenza che l'elettore utilizza quale principale criterio di scelta l'opinione sul partito e, possiamo aggiungere, negli ultimi tempi, la considerazione del leader del partito. L'effetto "figlio prediletto" ha perciò poca efficacia nel risultato elettorale dei singoli candidati e, conseguentemente, dei partiti. 
Con questa premessa, un'analisi in profondità sull'utilizzo del voto di preferenza può essere utile per stabilire se nello straordinario risultato della Lega nelle regioni meridionali abbiano influito quelle condizioni proprie di un partito che ha un suo radicamento territoriale, con attivisti che si mobilitano elettoralmente a favore dei candidati. Per fare questa verifica siamo andati a scandagliare cosa è successo dal punto di vista dell'espressione del voto di preferenza in quei comuni dove la Lega Salvini Premier ha ottenuto percentuali molto più alte della media regionale e circoscrizionale, riportando, di seguito, alcuni casi di micro-analisi comunale che ci aiutano a comprendere sia il comportamento degli elettori che l'effettiva presenza del partito sul territorio.

Partendo da nord a sud, riportiamo alcuni di questi casi. Si tratta quasi sempre di piccoli o piccolissimi comuni dove il voto di preferenza spesso si concentra su uno o pochissimi candidati. Nei comuni più grandi, invece, l'espressione del voto di preferenza è distribuita fra più candidati rendendo così più difficile, ai fini della nostra analisi, l'individuazione di uno o più candidati espressione del territorio.

In Abruzzo, e in tutto il Meridione, il comune che ha tributato la più alta percentuale di voti alla Lega è Civitella Roveto (AQ) con il 60,2\%. Nel 2018 la Lega, in questo comune, aveva ottenuto il $26,7 \%$. La candidata più votata è stata Elisabetta De Blasis con 681 voti di preferenza su 921 voti di lista, cioè 74 elettori su cento che hanno votato Lega hanno espresso una preferenza per questa candidata, medico e consigliere comunale dell'Aquila. Salvini in questo comune riceve solo 130 voti di preferenza (14\% sui voti di lista) nonostante fosse una donna ad essere la più votata e, quindi, per la regola sulla preferenza di genere, potenzialmente votabile in accoppiata con la De Blasis.

Nel Molise, dove la Lega passa dall'8,7\% della Camera 2018 al $24,3 \%$ delle Europee, l'elettore leghista fa un uso moderato del voto di preferenza (indice 15,6\%) con Salvini che riceve la preferenza dal $27 \%$ degli elettori che votano la sua lista. Non si registrano percentuali elevatissime nei comuni per la Lega Salvini Premier probabilmente perché non vi sono candidati del territorio in grado di fare man bassa dei voti (l'unico candidato molisano ha ricevuto solo il $6,6 \%$ di preferenze rispetto ai voti di lista).

La Campania, che fa registrare il livello più basso di consensi per la Lega, passa dal 4,3\% delle politiche al 19,2\% delle Europee. Anche in Campania i picchi per la Lega sono originati dal successo di uno o più candidati. Nel comune di Cassano Irpino (Av) la Lega, che nel 2018 era al $6,2 \%$, ottiene il $60,1 \%$ grazie, soprattutto, ai voti di preferenza ricevuti dai 
candidati Simona Sapignoli (224 voti di preferenza su 332 ottenuti dalla lista) e Valentino Grant (204 preferenze). Matteo Salvini in questo comune ottiene solo 10 voti di preferenza. A Roccagloriosa (Sa) la Lega passa dal $13,1 \%$ della Camera al $54,3 \%$ delle Europee con il contributo della candidata Lucia Vuolo che ottiene 265 preferenze su 376 voti di lista mentre Salvini ne ottiene solo 51. Pressappoco la stessa situazione che si verifica a Casalduni (Bn), con la Lega al 46,7\%, dove Nadia Sgro ottiene 249 voti di preferenza su 409 e il capolista Salvini 33.

In Puglia, a Zapponeta (Fg) la Lega passa dall'8,5\% del 2018 al $55,1 \%$ del 2019 e il merito di questo successo è da ascrivere sempre alla presenza di candidati in grado di ottenere voti personali. Andrea Caroppo ottiene 371 voti su 640 di lista, Massimo Casanova ne ottiene 176, Antonella Lella 144 mentre Matteo Salvini solo 26 preferenze. Andrea Caroppo, eletto al Parlamento Europeo, proviene da precedenti esperienze politiche, di consigliere regionale e consigliere comunale, con altri partiti. Un candidato, quindi, con radici profonde nel territorio. A Lesina (Fg), comune nel quale il candidato imprenditore Massimo Casanova ha una residenza, la Lega ottiene il 44,1\% dei consensi. Fra i 1.512 elettori che hanno votato Lega, 832 hanno espresso una preferenza per Casanova, mentre Salvini di preferenze ne ha ottenute 77.

La Lega in Basilicata alle regionali di due mesi prima era al 19,1\% e alle Europee ottiene il 23,3\% con un numero di voti praticamente identico fra le due elezioni (55.400 circa) ma con la percentuale diversa per via della minore partecipazione al voto alle Europee. Il comune lucano con la più alta percentuale alla Lega è Maratea $(\mathrm{Pz})$ con il 44,5\%. Un apporto a questo risultato è venuto senz'altro dalla candidata Daniela Calderano con 947 voti di preferenza su 1.309 voti di lista. Salvini riesce ad ottenere 174 voti di preferenza nonostante la possibilità per l'elettore di dare una seconda e terza preferenza ad un candidato uomo, dopo l'espressione del voto a favore della Calderano. Il successo di Daniela Calderano, di professione avvocato ma senza alcuna precedente esperienza politica, è circoscritta a Maratea e ai comuni limitrofi e non riesce ad andare oltre poiché la candidata non può disporre, per evidenti ragioni, di un proprio apparato e di attivisti in grado di coprire tutta la regione. La limitata presenza di rappresentanti sul territorio era stata evidenziata nelle regionali del marzo 2019 quando i candidati non avevano contribuito molto al successo della lista attraverso l'acquisizione di voti di preferenza (De Luca 2019). Complessivamente Matteo Salvini ottiene in Basilicata 16.484 voti di preferenza, quasi lo stesso numero (17.074) delle preferenze ottenute dagli altri 17 candidati. 
La Lega in Calabria passa dal 5,6\% della Camera 2018 al 22,6\% delle Europee. Oltre ai candidati calabresi, ottengono buoni risultati in fatto di preferenze candidati apparentemente lontani dalla Calabria ma, evidentemente, prossimi agli elettori calabresi tramite intercettori di consensi con la propensione all'effetto bandwagon. E soprattutto nei risultati di piccoli o piccolissimi comuni, dove il controllo sociale sul voto è più evidente, che possiamo rilevare il radicamento della nuova Lega di Salvini. Come ad esempio a San Pietro in Amantea (Cs) dove la Lega ottiene la maggioranza assoluta con il 59,7\%. Vincenzo Sofo ottiene 104 voti di preferenza su 132 voti di lista mentre Matteo Salvini di preferenze ne riceve solo 4. Il successo della Lega in questo piccolissimo comune è ascrivibile all'attivismo del sindaco che, all'indomani delle elezioni politiche del 2018, si è dichiarato leghista, e per le Europee ha condotto una campagna porta a porta fra i suoi concittadini. Sofo, milanese di nascita, compagno di Marion Le Pen, nipote della leader del Front National, è animatore di un laboratorio politico "sovranista" nonché amico di Matteo Salvini, l'unico legame che aveva con la Calabria, prima delle Europee, erano le origini dei suoi genitori. Questa sua distanza dalla Calabria e dalla circoscrizione meridionale, probabilmente, è stata colmata dal partito che ha suggerito agli attivisti sul territorio di "portare" il candidato Sofo. Un altro candidato sostenuto dal partito, che in Calabria poteva vantare accordi con esponenti di destra ${ }^{2}$, è Massimo Casanova. Un esempio del sostegno ottenuto da questi due candidati esterni lo abbiamo nel comune di Gizzeria (Cz) con la Lega che passa dall'11,3\% della Camera 2018 al 43,5\% delle Europee. I voti di lista sono 733 mentre Casanova è il più votato con 317 preferenze seguito da Sofo con 216 preferenze. Il capolista Salvini, anche lui esterno alla regione, ottiene, invece, 89 voti di preferenza.

Nelle regionali in Sicilia del novembre 2017, la Lega era stata presente con lo stesso simbolo di Fratelli d'Italia; insieme questi due partiti avevano ottenuto il 5,6\%, voti che avevano contribuito al successo della coalizione di centrodestra. Qualche mese dopo le regionali, la Lega alla Camera ottiene il 5,1\% e alle Europee il 20,8\%. Anche in Sicilia la presenza del candidato del territorio fa aumentare notevolmente i consensi per la lista. A Graniti (Me) la Lega Salvini Premier ottiene il 45,0\% dei voti alle Europee, partendo da un ottimo 37,3\% della Camera. In questo comune il più votato della Lega è Angelo Attaguile con 229 voti di preferenza su 313

\footnotetext{
${ }^{2}$ Un accordo importante siglato da Salvini è quello con Scopelliti, ex presidente della Regione Calabria, finito agli arresti per vicende collegate alla sua precedente attività di sindaco della città di Reggio Calabria.
} 
voti di lista, seguito da Matteo Salvini con 136 preferenze. Attaguile è un politico di lungo corso, ex DC, ex Movimento per le Autonomie di Raffaele Lombardo ex governatore della Sicilia con il quale viene eletto deputato nel 2013 iscrivendosi nel gruppo parlamentare della Lega Nord e Autonomie, indagato per voto di scambio prima della presentazione delle liste. Salvini ottiene numerosi consensi anche in altri comuni della Sicilia dove la Lega è il partito più votato. Significativo il risultato del comune di Lampedusa e Linosa $(\mathrm{Ag})$ con la Lega al 45,8\%, la più alta percentuale fra i comuni siciliani, e Salvini che ottiene 410 preferenze su 624 voti di lista. In questo comune ottiene un buon risultato il PD e il candidato Pietro Bartolo (250 preferenze su 285 voti di lista), il "medico di Lampedusa" del centro di accoglienza immigrati. In tutta la Sicilia Salvini viene votato da circa il $57 \%$ di quanti esprimono una preferenza per la Lega e complessivamente quasi il $60 \%$ dei voti di preferenza dei candidati della Lega.

In Sardegna la Lega, partendo dal buon risultato delle regionali del febbraio 2019 (11,4\%) e dagli accordi, ufficiali e non, con gruppi e movimenti autonomisti, ottiene il 27,6\% alle Europee. 43 elettori su 100 della Lega in Sardegna esprimono una preferenza per Matteo Salvini che raccoglie complessivamente il 53\% dei voti di preferenza espressi per i candidati in lista. A Tortolì $(\mathrm{Nu})$ la Lega ottiene il 45,0\% dei consensi grazie al candidato del luogo Massimiliano Piu, 1.273 preferenze su 1.705 voti di lista e con Salvini che, comunque, ottiene 444 voti. A Cabras (Or) la Lega è al $43,1 \%$ con 1.101 voti e 494 preferenze per Matteo Salvini e con i candidati della regione che seguono con 171 preferenze Sonia Pilli e 167 Massimiliano Piu. La circostanza che Salvini sia votato nelle preferenze molto più rispetto ai due soli candidati della regione confermerebbe l'ipotesi che il criterio maggiormente utilizzato dagli elettori sardi sia l'opinione nei confronti della persona leader del partito.

7. Il valore aggiunto dei candidati alla performance di altri partiti: alcuni casi esemplari al Sud

I casi che abbiamo esaminato riguardano il valore aggiunto dei candidati al successo della Lega in una competizione che, come abbiamo detto in precedenza, quale conseguenza della vastità della circoscrizione, non consente una vicinanza fra candidati ed elettori. L'apporto dei candidati alla lista è un elemento comune a quasi tutti i partiti in competizione ed il voto personale al candidato è anche una caratteristica molto comune nel comportamento elettorale dei meridionali (Bagnasco, Piselli, Pizzorno, 
Trigilia 2001; Putnam, Leonardi, Nanetti 1993). Per la conquista di consensi personali, il candidato interviene direttamente nella campagna elettorale e, soprattutto quando sono molti gli elettori interessati al voto, come in questo caso, intervengono attivisti e sostenitori del partito e/o del candidato. Fra i principali partiti, il M5S viene penalizzato nei sistemi proporzionali con il voto di preferenza, soprattutto a causa della modalità di selezione e delle caratteristiche dei candidati (senza precedenti esperienze, poco noti, giovani, ecc.). Alla luce dei risultati di elezioni con sistemi proporzionali e voto di preferenza (comunali e regionali, soprattutto), possiamo affermare che il "non-partito" M5S presenta liste di "non-candidati", cioè candidati che non sono in grado di far convergere sulla propria persona un consistente numero di voti di preferenza.

Di seguito riportiamo altri casi di comuni delle regioni meridionali nei quali l'ottimo risultato di una lista è da attribuire allo straordinario successo di candidati. Quasi sempre a raggiungere un risultato fuori dalla norma è un candidato del luogo il quale riceve voti a prescindere dal simbolo sotto il quale si presenta. Addirittura può accadere che in un piccolissimo comune, Calanna in provincia di Reggio Calabria, il primo partito sia Casapound, formazione che si richiama al fascismo, con il 32,7\% dei voti. Nella lista Casapound, Luigi Catalano ottiene 95 preferenze su 98 voti di lista. Catalano era stato sindaco di Calanna dal 2007 al 2012 e, evidentemente, ha mantenuto un rapporto politico fiduciario con almeno un terzo dei suoi concittadini che lo hanno votato senza porsi alcuna obiezione circa il simbolo sotto al quale si presentava l'ex-sindaco.

A Capracotta (Is) il PD raggiunge il 46,2\% dei consensi grazie alla giovane candidata molisana Caterina Cerroni che ottiene 127 preferenze su 163 voti andati al partito. In Campania, nel comune di Pignataro Maggiore (Ce), Forza Italia ottiene il 57,8\%. Il merito di questo eccellente risultato è tutto da attribuire al candidato Giorgio Magliocca, sindaco dello stesso comune di Pignataro Maggiore e presidente della Provincia di Caserta, che ottiene 1.482 voti di preferenza sui 1.569 della lista.

A Maglie (Le), Fratelli d'Italia è il primo partito con 1.981 voti e il 29,6\%. Raffaele Fitto, già presidente della Regione Puglia dal 2000 al 2005 e ministro dal 2008 al 2011 con presidente del Consiglio Silvio Berlusconi, riceve 1.795 voti di preferenza. Maglie è la città natale di Fitto ed è quindi "naturale" che riceva un così alto numero di consensi, così come erano moltissimi i voti che otteneva il partito con il quale si presentava alle elezioni. Fitto è stato democristiano (il padre di Raffaele è stato un politico di rilievo della DC in Puglia), poi PPI, CdU, Forza Italia, PdL, nel 2015 ha fondato un partito, Conservatori e Riformisti, in polemica con Forza Italia e 
nel 2017 fonda e diventa presidente di Direzione Italia, una formazione politica che parteciperà alle elezioni prima con Noi con l'Italia-UDC e nelle elezioni regionali ed Europee del 2019 con Fratelli d'Italia. I tanti cambiamenti di casacca non hanno influito sulla raccolta del consenso e sulla fiducia degli elettori nei confronti di Raffaele Fitto, a prescindere dal simbolo sotto il quale si è via via presentato. La sua precedente attività di governatore regionale, di ministro e di parlamentare nazionale ed europeo ha rafforzato i rapporti con alcuni «grandi elettori» ${ }^{3}$. I risultati ottenuti da Fratelli d'Italia e da Fitto in queste Europee attestano la vasta rete del consenso personale. Come, ad esempio, a Candela (Fg) con Fratelli d'Italia al $25,0 \%$ e Fitto con 225 voti di preferenza su 288 voti di lista.

Nei comuni dove la Lega ottiene una bassa percentuale, il risvolto della medaglia quasi sempre è un partito che riceve un forte consenso per l'impulso dato da qualche candidato tramite la conquista di voti di preferenza. È senz'altro così a Francavilla in Sinni $(\mathrm{Pz})$ con la Lega all' $11,1 \%$ e Forza Italia al $35,2 \%$ e la giovane candidata campana Antonella Pecchia che ottiene 285 voti di preferenza su 476 voti di lista e con il capolista Berlusconi che ne ottiene 277. È così anche nel popoloso comune di Avola (Sr) con la Lega all'8,8\% e Fratelli d'Italia al 54,9\%, con il candidato, figlio prediletto della città e suo sindaco, Luca Cannata che riceve 5.872 voti di preferenza su 6.058 voti di lista, cioè 97 elettori su 100 che hanno votato per Fratelli d'Italia hanno espresso una preferenza per Cannata. Ma sarebbe più corretto dire che una buona parte di quei 6.058 cittadini di Avola hanno votato solo per Luca Cannata.

La Calabria offre alcuni interessanti spunti di riflessione sull'utilizzo del voto di preferenza. A Laureana di Borrello (Rc) Fratelli d'Italia è al $56,9 \%$ grazie all'apporto del candidato del luogo Denis Nesci che riceve 1.366 voti di preferenza su 1.484 voti di lista. Sempre lo stesso candidato porta al successo la propria lista a Platì (Rc) con il 48,5\%. Nesci, in questo comune, ottiene 324 voti di preferenza su 325 voti di lista! Un risultato che ha dell'incredibile e induce a pensare ad un possibile refuso nella trascrizione del dato. A Capistrano (Vv) Forza Italia raggiunge il 59,2\% con 299 voti. Il più votato della lista non è un candidato calabrese ma è Lorenzo Cesa - romano, più volte deputato nel parlamento italiano ed europeo e

\footnotetext{
${ }^{3}$ Per «grandi elettori» intendiamo coloro che, sfruttando uno status quasi permanente di amministratore locale o ricoprendo ruoli importanti nella pubblica amministrazione o in importanti soggetti economici privati, sono in grado di svolgere una serrata campagna elettorale a favore di un candidato. Per una più ampia definizione, si veda De Luca (2001).
} 
segretario dell'Unione di Centro - con 265 voti e con il capolista Silvio Berlusconi che ottiene un solo voto di preferenza.

\section{Conclusioni - Un voto debole?}

L'avanzamento della Lega di Salvini nel Mezzogiorno alle ultime elezioni europee è stato significativo. Il risultato è plateale e clamoroso perché la Lega è diventato nel Mezzogiorno il secondo partito dopo il M5S. Eppure alle precedenti elezioni europee aveva sfiorato 1 ' $1 \%$ e alle elezioni politiche di un anno prima aveva ottenuto poco più del $6 \%$ dei voti. Molti osservatori hanno attribuito quel successo all'appeal di Salvini e alla sua efficace campagna elettorale. Si tratta di una ipotesi plausibile e condivisa anche da noi. Tuttavia, in questo contributo abbiamo cercato di valutare se la misura del successo della Lega sia stata più consistente anche grazie al contributo dei candidati o dei loro sostenitori, ben insediati nelle comunità locali, che recentemente hanno aderito alla Lega.

Tale interrogativo deve essere collocato nella questione più generale del cambiamento organizzativo della Lega. In questo articolo non ci siamo occupati del ri-orientamento programmatico della Lega. Su tale argomento, per altro, il dibattito è piuttosto vivace. Noi abbiamo focalizzato l'attenzione sul ruolo dei candidati alle elezioni europee in base al presupposto che le modalità attraverso cui si seleziona il personale politico rappresentino un importante aspetto della struttura organizzativa di un partito. La strategia di insediamento nel Mezzogiorno è diversa da quella che la Lega ha sperimentato nelle regioni settentrionali in cui ormai è il partito più votato.

Al Nord la Lega si era insediata nelle comunità locali, sollecitando partecipazione e senso di appartenenza. Negli ultimi anni il tessuto associativo della Lega si è indebolito. Tuttavia nel frattempo la Lega è riuscita a dotarsi di un ceto politico formatosi attraverso l'esperienza di governo nelle istituzioni locali che è in grado di affrontare con successo la competizione elettorale. Anche la Lega, seguendo la parabola di altri partiti tradizionali, ha spostato il proprio baricentro dalla società alle istituzioni. La lega ha adottato una strategia di insediamento organizzativo al sud puntando sul reclutamento di personale politico, non sempre originale, spesso riciclato. Si tratta di personalità che in qualche caso hanno un cursus honorum in politica, che hanno dimestichezza con i meccanismi di raccolta del consenso e che rappresentano perciò una risorsa nella competizione elettorale. 
Di conseguenza, la nostra analisi ha cercato di valutare il contributo dei candidati nella raccolta del consenso e dunque nella definizione del patrimonio elettorale complessivo della Lega. Ebbene sulla scorta dell'analisi della distribuzione del voto di preferenza abbiamo rilevato che in alcuni contesti, in particolare nei piccoli comuni sono candidati diversi dal capolista Salvini a raccogliere il maggior numero di consensi personali. In effetti, mentre al nord Salvini è il candidato più votato in quasi tutti i comuni, al sud la percentuale di comuni in cui Salvini è il candidato più votato è più bassa soprattutto con riferimento ai centri delle regioni meridionali aventi una popolazione inferiore a 15 mila abitanti. Analogamente, il tasso di leadership ossia la quota di preferenze ottenuta da Salvini sul totale delle preferenze raccolte da tutti i candidati è addirittura al di sotto del 50\% nei comuni del sud aventi meno di 15 mila abitanti.

Alle medesime conclusioni siamo pervenuti focalizzando l'osservazione su alcuni piccoli comuni in cui la Lega ha ottenuto le migliori performance elettorali. Emerge il ruolo svolto dagli altri candidati che hanno beneficiato del supporto di notabili locali attratti anch'essi nell'orbita del partito del Vice Presidente del Consiglio e Ministro dell'Interno, Matteo Salvini.

Sulla scorta di questi risultati è possibile svolgere alcune considerazioni che hanno per tema la forza o la debolezza del risultato della Lega.

Le consultazioni elettorali più recenti in molti paesi sono segnate da una elevata volatilità. $E$ anche nel nostro paese i risultati variano in misura considerevole di volta in volta in esito a scelte connesse ad elementi contingenti della proposta politica. Anche il successo della Lega nelle regioni meridionali, dunque, potrebbe essere definito debole. La fedeltà al partito e, conseguentemente, il voto d'appartenenza - in verità poco diffuso nel Mezzogiorno - sembrano essere definitivamente tramontati.

L'elettore meridionale ha effettuato la propria scelta di voto per una duplice influenza: da lontano e da vicino. L'influenza da lontano è quella legata al leader alle sue capacità oratorie e comunicative, più in generale. La forza di questa influenza da lontano deriva anche dall'abilità del leader di organizzare una macchina di propaganda efficace. L'influenza da vicino è quella della richiesta del voto alla persona nel porta a porta messo in atto da attivisti e carrieristi secondo l'accezione proposta da Panebianco (1982). Si tratta di personale politico professionale o semiprofessionale che attraverso la partecipazione alla politica locale dispone di un certo numero di consensi personali. Salvini ha cercato di mettere radici in un territorio finora da lui inesplorato, alquanto ostile, dando fiducia a questo tipo di personale politico. 
Il ruolo del leader potrebbe rappresentare un elemento di debolezza del consenso alla Lega. Le vicende politiche del nostro paese mostrano che la cronaca politica recente è un cimitero di leader la cui parabola politica si è rapidamente esaurita. Al contrario lo sviluppo organizzativo dovrebbe consentire il consolidamento del consenso al partito. Tuttavia sia la costruzione della macchina della propaganda che le modalità del reclutamento del personale politico propongono rischi in vista della stabilizzazione del consenso alla Lega.

In primo luogo la macchina della comunicazione sembra troppo aderente alle caratteristiche del leader e dunque si espone ad una rapida obsolescenza se il leader fallisce. Più controverse sono le conseguenze delle modalità di reclutamento del personale politico.

L'analisi sulla distribuzione del voto di preferenza suggerisce una partecipazione del personale politico locale al patrimonio elettorale della Lega. Tale risultato sembra valorizzare il ruolo nella competizione elettorale dei notabili locali. Questo risultato sembra in contraddizione con il risultato del M5S nel 2018 che aveva fatto della polemica contro i politici professionali la propria bandiera. Tuttavia anche alle elezioni del 2018 era emerso che i candidati dei partiti tradizionali nei collegi uninominali erano riusciti ad ottenere un surplus di voti rispetto ai consensi raccolti dalle liste (Fruncillo e Giannatiempo 2018). D'altro canto, il consistente calo di consensi al M5S anche alle elezioni europee potrebbe risiedere proprio nella circostanza che quel partito al momento non dispone di personale politico locale.

Tuttavia, se si tiene conto delle modalità con cui sta provando ad insediarsi al Sud, la Lega rischia di finire ostaggio degli eletti e dei candidati. Il personale politico che ha accolto l'invito della Lega persegue un progetto autonomo. La nostra ipotesi è che molti candidati abbiano aderito alla Lega per ricavare i vantaggi che potrebbero derivare dal rapporto preferenziale con il governo nazionale, attraverso la Lega. Si tratta di personaggi politici che sperano di ottenere dal governo nazionale risorse materiali e simboliche per rafforzare la loro capacità di raccogliere consensi. Se questo non dovesse accadere non è improbabile che essi veleggino verso altri approdi depotenziando le risorse elettorali della Lega.

In estrema sintesi, la nostra analisi ha mostrato che la Lega ha, ormai, un buon radicamento elettorale anche nelle regioni meridionali e che esso potrebbe rafforzarsi grazie ad una sorta di effetto band-wagon per cui molti politici professionali o semiprofessionali carrieristi sono attratti dalla corte di Salvini. Naturalmente questo effetto potrebbe rafforzarsi a prescindere dall'attuale partecipazione della Lega all'Esecutivo nazionale 
perché essa si sta accreditando come un partito destinato, prima o poi, al governo del paese. Tuttavia, la posizione della Lega al sud diventerebbe più debole anche sul piano elettorale se i suoi competitori riuscissero a mostrare le contraddizioni programmatiche di un partito che chiede consensi ai cittadini meridionali pur continuando ad avere solide radici al nord come mostrano alcune proposte tra le quali la cosiddetta autonomia regionale differenziata. 


\section{Riferimenti bibliografici}

A. Bagnasco, Piselli F., Pizzorno A. e Trigilia A. (2001), Il capitale sociale. Istruzioni per l'uso, Il Mulino, Bologna.

M. Balmas, Rhat, G., Sheafer, T., e Shenhav, S. R. (2014), «Two routes to personalized politics: Centralized and decentralized personalization», Party Politics, 20 (1), pp. 37-51.

S. Bartolini (1996), «Partiti politici e sistemi di Partito» in Enciclopedia della Scienze Sociali, Torino, UTET, pp. 616-532.

S. Bartolini P. Mair (2001), Challenges to Contemporary Political Parties in R. Gunther e L. Diamond (eds) ... pp. 323-343.

M. Brunazzo e C. Roux (2013), The Lega Nord: From Regional Protest to National Government, Paper presentato alla 7th ECPR General Conference, Bordeaux, 4-7 settembre 2013 (Section 30 - New Developments in Federalism and Regionalism Panel 282 - Regionalist Parties in National Institutions).

M. Calise (a cura di) (1992), Come cambiano i partiti, Il Mulino Bologna.

R. J. Dalton, M. P. Wattenberg, (a cura di) (2000), Parties Without Partisan. Political Change in Advanced Industrial Democracies, Oxford, Oxford University Press.

R. De Luca (2001), «Il ritorno dei "campioni delle preferenze" nelle elezioni regionali», in Polis, 15 (2), pp.227-248.

$\mathrm{R}$. De Luca (2013), Le liste non partitiche e lo smisurato ampliamento dell'offerta nelle elezioni comunali, in Diamanti I, Ceccarini L. (a cura di), Sondaggi ed elezioni. Le regole del gioco e della comunicazione, SISE, Firenze, pp. 298-327.

R. De Luca (2018), Dove ha contato (ancora) il voto alla persona. Il voto nei collegi meridionali vinti (o quasi) dal centrodestra, in D. Fruncillo e F. Addeo (a cura di), Le elezioni del 2018. Partiti, candidati, regole $e$ risultati, Società Italiana di Studi Elettorali, Firenze, pp. 34-49.

R. De Luca (2019), «La vittoria del "partito nazionale" di Salvini», in www.rivistailmulino.it, 27 marzo.

I. Diamanti (1993), La Lega. Geografia, storia e politica di un nuovo soggetto politico, Roma, Donzelli editore.

I. Diamanti (2003), Bianco rosso verde e ... azzurro, Bologna, Il Mulino. 
I. Diamanti (2009), Mappe dell'Italia politica. Bianco, rosso, verde, azurro ... e tricolore, Bologna il Mulino.

M. Duverger (1961), I partiti politici, Milano, Edizioni di Comunità.

D. Fruncillo (2006), «Perifericità sociale e marginalità politica. Una proposta di analisi del risultato delle elezioni politiche del 2006», in Democrazia e Diritto, Vol. 4, pp. 159-183.

D. Fruncillo (2016), «La "mobilitazione" personale e la partecipazione alle elezioni regionali in Italia», in Quaderni dell'Osservatorio elettorale, Vol. 75, pp. 37-82.

D. Fruncillo (2018), «Il voto al Sud», in Note e Comunicati, Istituto Cattaneo, 12 aprile, www.cattaneo.org

D. Fruncillo, M. Giannatiempo (2018), Personalizzazione $e$ antipolitica. La competizione nei collegi uninominali alle elezioni del 2018, in D. Fruncillo e F. Addeo (a cura di), Le elezioni del 2018. Partiti, candidati, regole e risultati, Società Italiana di Studi Elettorali, Firenze, pp. 8-33.

R. Katz e P. Mair (eds.) (1994), How Parties Organize: Adaption and Change in Party Organisations in Western Democracies, Londra, Sage.

V. O. Key1(964), Politics, Parties and Pressure Groups, New York, Crowell.

O. Kirchheimer (1966), The Transformation of the Western European Party System, in J. La Palombara, M. Weiner (eds.), Political Parties and Political Development, Princeton University Press, Princeton, pp. 177-200 [trad. it. Le trasformazioni dei sistemi partitici dell'Europa occidentale in G. Sivini (a cura di) (1979), Sociologia dei partiti politici. Le trasformazioni nelle democrazie rappresentative, Bologna, Il Mulino pp.243-269].

P. Mair (1992), La trasformazione del partito di massa in Europa, in M. Calise (a cura di), Come cambiano i partiti, cit., pp. 99-120.

E. Melchionda (a cura di) (1996), Il destino dei partiti, Ediesse Roma.

A. Panebianco, 1982, Modelli di partito, Bologna, Il Mulino.

G. Passarelli, D. Tuorto (2012), Lega \& Padania. Storie e luoghi delle camicie verdi, Bologna, Il Mulino.

G. Passarelli, D. Tuorto (2018), La Lega di Salvini. Estrema destra di governo, Il Mulino, Bologna.

R.D. Putnam, Leonardi R. e Nanetti R.Y. (1993), Making Democracy Work: Civic Traditions in Modern Italy, Princeton University Press, Princeton.

A. Signore, A. Trocino (2008), Razza Padana, BUR, Milano. 
M. Valbruzzi (2019), «Elezioni regionali in Sardegna 2019: Chi ha vinto, chi ha perso», in Note e Comunicati, Istituto Cattaneo, 26 febbraio, www.cattaneo.org

L. Verzichelli (2010), Vivere di politica: come (non) cambiano le carriere politiche in Italia, Il Mulino, Bologna. 